\title{
Tetraquark-Adequate QCD Sum Rules
}

\section{Wolfgang Lucha*}

Institute for High Energy Physics, Austrian Academy of Sciences, Nikolsdorfergasse 18, A-1050 Vienna, Austria

E-mail: Wolfgang. Lucha@oeaw.ac.at

\section{Dmitri Melikhov}

Institute for High Energy Physics, Austrian Academy of Sciences, Nikolsdorfergasse 18, A-1050 Vienna, Austria, and

D. V. Skobeltsyn Institute of Nuclear Physics, M. V. Lomonosov Moscow State University, 119991, Moscow, Russia, and

Faculty of Physics, University of Vienna, Boltzmanngasse 5, A-1090 Vienna, Austria

E-mail: dmitri_melikhov@gmx.de

\section{Hagop Sazdjian}

Institut de Physique Nucléaire, CNRS-IN2P3, Université Paris-Sud, Université Paris-Saclay, 91405 Orsay Cedex, France

E-mail: sazdjian@ipno.in2p3.fr

\begin{abstract}
With the experimental observation of several credible candidates for multiquark hadrons, the latter states re-entered the focus of interest of theoretical strong-interaction physics. Proper treatment of hadronic bound states by quantum chromodynamics, QCD, the quantum field theory governing all strong interactions, necessitates a nonperturbative approach. A well-established framework of this kind is provided by QCD sum rules relating hadron features to the parameters of QCD. Conceptual reconsideration, however, reveals that, in order to really match the peculiarities of multiquarks, the long-standing conventional QCD sum-rule techniques evidently must be subjected to considerable modification. The so far overlooked necessity for such adaptations is most easily demonstrated for the case of least complexity, that is, for tetraquarks, bound states of two quarks and two antiquarks.
\end{abstract}

European Physical Society Conference on High Energy Physics - EPS-HEP2019

10-17 July, 2019

Ghent, Belgium

\footnotetext{
* Speaker.
} 


\section{Preliminaries: Setting the Theater of Multiquark QCD Sum-Rule Considerations}

The quantum field theory of strong interactions, quantum chromodynamics, enables as possible (colour-singlet) bound states of quarks and gluons not just quark-antiquark mesons and three-quark baryons, usually subsumed by the notion ordinary hadrons, but also multiquark hadrons, sometimes dubbed exotic. For the latter species, we seek trustworthy descriptions by means of QCD sum rules.

QCD sum rules [1] constitute a nonperturbative approach to bound states of quarks and gluons, the basic degrees of freedom of QCD, in form of analytic relations between observable properties of hadrons, on the one hand, and the parameters of QCD, i.e., strong coupling and quark masses, on the other hand. Usually, they are distilled by evaluation of correlation functions of hadron interpolating operators defined in terms of quark and gluon fields at both phenomenological (hadronic) and $Q C D$ levels: by insertion of a complete set of hadron states, conversion of nonlocal operator products into series of local operators by use of Wilson's [2] operator product expansion, removal of any required subtraction terms and suppression of the hadron contributions above the ground state by performing Borel transformations, and relying on the assumption that all perturbative QCD contributions above Borel-variable governed [3-5] effective thresholds cancel against hadron continuum. At QCD level, the relationships receive both purely perturbative contributions, conveniently represented in form of dispersion integrals of spectral densities, and nonperturbative contributions involving QCD vacuum condensates multiplied by powers of Borel variables and therefore being dubbed power corrections.

Before adopting QCD sum-rule techniques for extracting information on the basic properties of multiquark states, such as tetraquarks and pentaquarks, two issues have to be settled: an, or even the most, suitable choice of interpolating operators and the formulation of a selection criterion ensuring an unambiguous identification of all relevant QCD contributions to the correlators considered [6,7].

In terms of quark flavour quantum numbers $a, b, c, d \in\{u, d, s, c, b\}$, a tetraquark $T$ is a mesonic bound state $\left(\bar{q}_{a} q_{b} \bar{q}_{c} q_{d}\right)$ of two quarks $q_{b}, q_{d}$ and two antiquarks $\bar{q}_{a}, \bar{q}_{c}$, with masses $m_{a}, m_{b}, m_{c}, m_{d}$. The colour degree of freedom of the (anti-) quarks, transforming according to the three-dimensional (anti-) fundamental representation of the gauge group SU(3) underlying QCD, does not matter for a trivial reason. Labelling each representation by its dimension, one notices the appearance of merely two SU(3) singlet representations in the tensor product of two 3 and two $\overline{3}$ representations of SU(3):

$$
3 \otimes 3 \otimes \overline{3} \otimes \overline{3}=81=\mathbf{1} \oplus \mathbf{1} \oplus 8 \oplus 8 \oplus 8 \oplus 8 \oplus 10 \oplus \overline{\mathbf{1 0}} \oplus 27 .
$$

It is easy to demonstrate that, irrespective of the route followed in the formation of each of these two colour singlets in intermediate steps, by application of Fierz transformations the arising operators of two-quark-two-antiquark form can be recast into the shape of known sums of products of colourless quark-antiquark bilinear operators. In view of these observations, for the construction of tetraquark interpolating operators it suffices to utilize, as local building blocks, colour-singlet quark-antiquark bilinear currents of (if suppressing possible but for the following irrelevant Dirac structures) generic shape $j_{\bar{a} b}(x) \equiv \bar{q}_{a}(x) q_{b}(x)$. With these, no more than only two tetraquark interpolating operators of current-current form are conceivable, namely, $\theta_{\bar{a} b \bar{c} d}(x) \equiv j_{\bar{a} b}(x) j_{\bar{c} d}(x)$ and $\theta_{\bar{a} d \bar{c} b}(x) \equiv j_{\bar{a} d}(x) j_{\bar{c} b}(x)$.

Since the quark content of a tetraquark may likewise (or preferably) form two ordinary mesons, we use sharp blades. Presumptive QCD support of a tetraquark pole is called tetraquark-phile $[8,9]$ :

The set of all tetraquark-phile Feynman diagrams is straightforwardly characterized [6] by the behaviour of each member as function of the appropriate Mandelstam variable $s$ : 
any member has to depend nonpolynomially, i.e., nontrivially, on $s$ and to enable one or more genuine four-quark intermediate states by exhibiting branch cuts (the existence of which can be verified by reverting on Landau's equations [10]) starting at branch points defined by the masses of the bound-state constituents, i.e., at $s=\left(m_{a}+m_{b}+m_{c}+m_{d}\right)^{2}$.

\section{Brief Line of Argument: Tetraquark Characteristics from Two-Point Correlators}

Given a tetraquark $T$, we intend to derive its basic features, i.e., its mass $M$ and decay constants

$$
f_{\bar{a} b \bar{c} d} \equiv\left\langle 0\left|\theta_{\bar{a} b \bar{c} d}\right| T\right\rangle \quad \text { and } \quad f_{\bar{a} d \bar{c} b} \equiv\left\langle 0\left|\theta_{\bar{a} d \bar{c} b}\right| T\right\rangle,
$$

from its pole contributions to two-point correlators of appropriate operators $\theta$, by formulating QCD sum rules which take into account the nonconventional nature of multiquarks [11]. For definiteness, let us sketch our reasoning for the case of tetraquarks involving four different quark flavours. There, we better discriminate two types of contributions to the tetraquark poles, namely, flavour-preserving and flavour-rearranging ones, emerging from adopting two interpolating operators of either equal or unequal quark flavour distributions. When evaluating some correlator at QCD level, its perturbative contributions will emerge in form of series expansions in powers of the strong coupling $\alpha_{\mathrm{s}} \equiv g_{\mathrm{s}}^{2} / 4 \pi$.

Figure 1 recalls the quark-bilinear origin of flavour-retaining correlators at lowest orders of $\alpha_{\mathrm{s}}$.
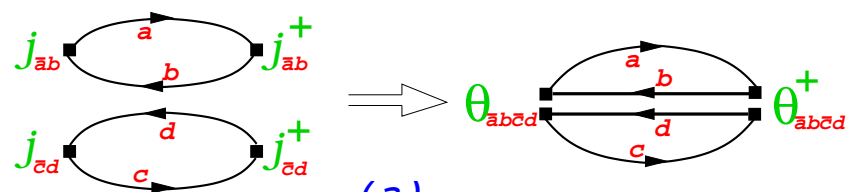

(a)
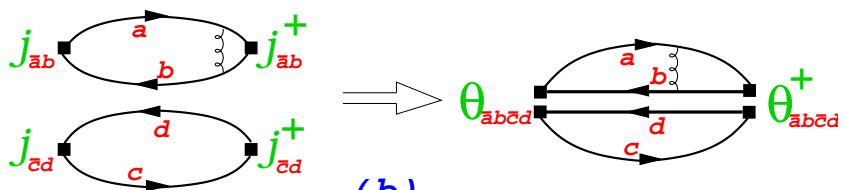

(b)
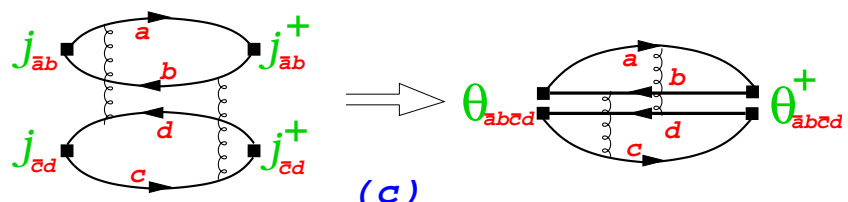

(c)

Figure 1: Flavour-preserving Feynman diagrams contributing at strong-coupling order $O\left(\alpha_{\mathrm{s}}^{0}\right)$ (a), $O\left(\alpha_{\mathrm{s}}\right)$ (b), and $O\left(\alpha_{\mathrm{s}}^{2}\right)$ (c) to correlators of four quark-bilinear currents $j$ (left) as well as, by exerting configuration-space pair contraction of quark-bilinear currents $j$, to correlators of two tetraquark interpolating operators $\theta$ (right).

Now, Feynman diagrams of order less than $O\left(\alpha_{\mathrm{s}}^{2}\right)$ do not comply with the criteria necessary for deeming them tetraquark-phile: Those of $O\left(\alpha_{\mathrm{s}}^{0}\right)$ [e.g., Fig. 1(a)] and those of $O\left(\alpha_{\mathrm{s}}\right)$ [e.g., Fig. 1(b)] with a single gluon (indicated by curly black lines) exchanged inside a quark loop contribute only to two ordinary mesons. All those of $O\left(\alpha_{\mathrm{s}}\right)$ with a single gluon exchanged between the two, otherwise disconnected quark loops are proportional to the definitely vanishing traces of all $\mathrm{SU}(3)$ generators. Hence, only at $O\left(\alpha_{\mathrm{s}}^{2}\right)$ [e.g., Fig. 1(c)] or higher Feynman diagrams start to contribute to tetraquarks.

Feeding the QCD sum-rule machinery with the inferred correlators, without paying attention to 
multiquark peculiarities, yields relations between QCD and hadron (identified by dashed blue lines) representations generically involving not only connected contributions but also not tetraquark-phile contributions separable (illustrated by a dot-dashed red line) into two unconnected portions (Fig. 2). Each of these two unconnected portions forms, however, the QCD sum rule for the correlator of two quark-bilinear currents $j$, i.e., for an ordinary meson (Fig. 3): a lucky circumstance that enforces the exact cancellation of all unconnected QCD and hadron contributions. That simple observation [11], if and only if taken into account, yields an adequate QCD sum-rule approach to tetraquarks (Fig. 4).

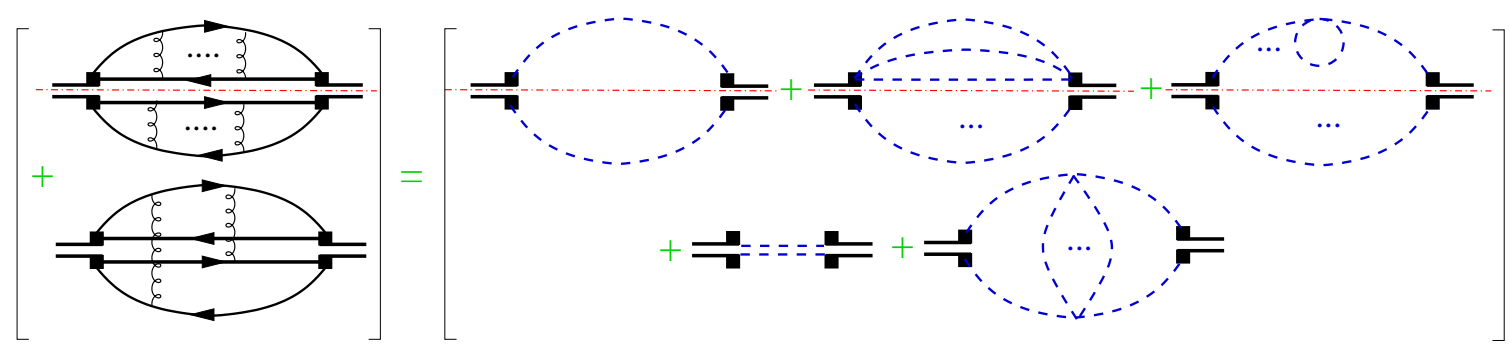

OPE side of $S R$

Hadron side of $S R$

Figure 2: Diagrammatic QCD sum rules emerging from correlators of two tetraquark interpolating operators $\theta$, as a consequence of the unreflecting observance of conventional recipes exhibiting on both QCD (left) and hadron (right) sides unconnected (top, separated by dot-dashed red lines) as well as connected (bottom) parts.

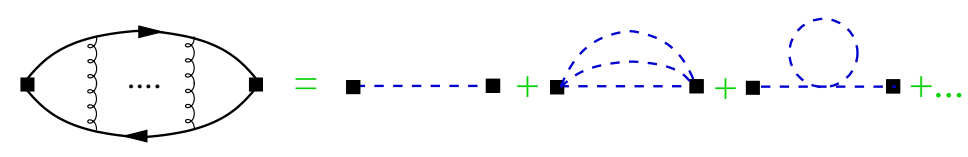

Figure 3: Diagrammatic QCD sum rules for ordinary mesons from correlators of two quark-bilinear currents $j$, contributing twice (on both sides of those separating dot-dashed red lines) to the unconnected part of Fig. 2.

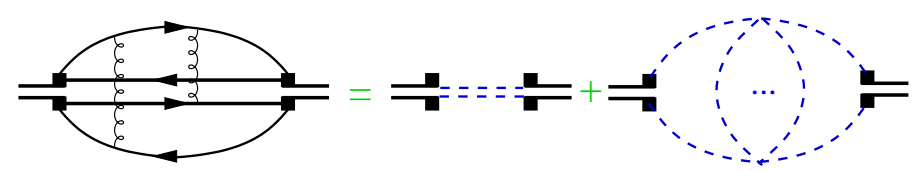

Figure 4: Diagrammatic QCD sum rules tailored to the adequate description of tetraquarks by factorizing off twice the QCD sum rules for ordinary mesons of Fig. 3 from the (before never challenged) relations of Fig. 2.

Figure 5 exemplifies contributions of lowest orders in $\alpha_{\mathrm{s}}$ to the flavour-reordering correlator of two not identical tetraquark currents $\theta$ formed by merging two quark bilinears: here, we cannot take advantage of some cancellation. However, application of the Landau equations [10] reveals that any contributions of orders $O\left(\alpha_{\mathrm{s}}^{0}\right)$ [Fig. 5(a)] or $O\left(\alpha_{\mathrm{s}}\right)$ [Fig. 5(b)] cannot support a tetraquark pole: only Feynman diagrams of order $O\left(\alpha_{\mathrm{s}}^{2}\right)$ [Fig. 5(c)] or higher may be considered as tetraquark-phile [6,7].

Following this line of argument and implementing the insights gained, one ends up with a novel kind of $Q C D$ sum rules, tailored to the requirements of tetraquark analyses, of the generic shape [11]

$$
\begin{aligned}
&\left(f_{\bar{a} b \bar{c} d}\right)^{2} \exp \left(-M^{2} \tau\right)=\int_{\left(m_{a}+m_{b}+m_{c}+m_{d}\right)^{2}}^{s_{\mathrm{eff}}} \mathrm{d} \exp (-s \tau)+\text { power corrections }, \\
& f_{\bar{a} b \bar{c} d} f_{\bar{a} d \bar{c} b} \exp \left(-M^{2} \tau\right)=\int_{\left(m_{a}+m_{b}+m_{c}+m_{d}\right)^{2}}^{s_{\mathrm{eff}}} \rho_{\mathrm{r}}(s)+\text { power corrections } \\
& \mathrm{d} \exp (-s)
\end{aligned}
$$



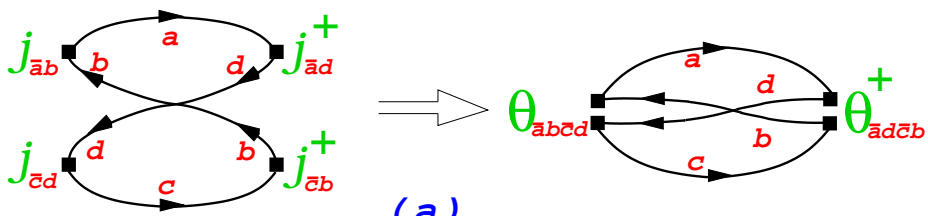

(a)
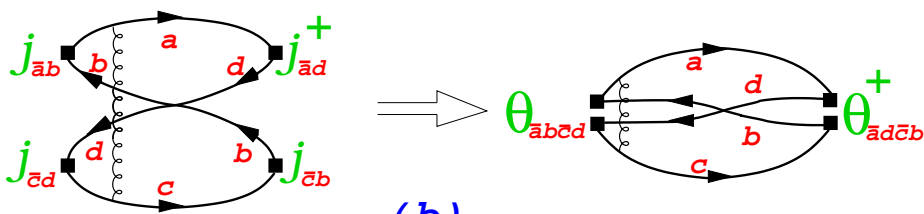

(b)
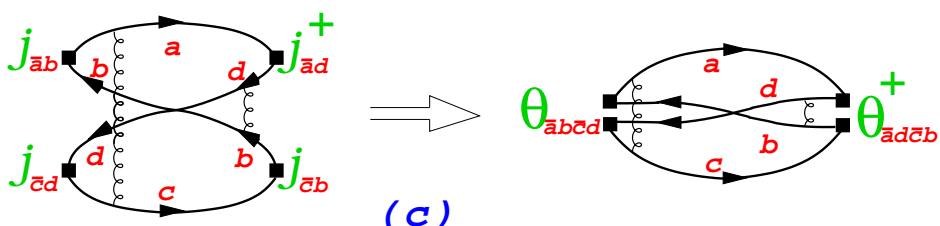

Figure 5: Flavour-reordering Feynman diagrams contributing at strong-coupling order $O\left(\alpha_{\mathrm{s}}^{0}\right)$ (a), $O\left(\alpha_{\mathrm{s}}\right)$ (b), and $O\left(\alpha_{\mathrm{s}}^{2}\right)$ (c) to correlators of four quark-bilinear currents $j$ (left) as well as, by exerting configuration-space pair contraction of quark-bilinear currents $j$, to correlators of two tetraquark interpolating operators $\theta$ (right).

involving the variable $\tau$ introduced by Borel transformation, $\tau$-dependent [3-5] effective thresholds $s_{\text {eff }}$, and spectral densities $\rho_{\mathrm{p}, \mathrm{r}}$ in flavour-preserving and flavour-rearranging instances, governed (as perforce also the power corrections) by exclusively tetraquark-phile contributions to the correlators.

\section{Outcome: Traditional Formulations of QCD Sum Rules Require Reconsideration}

Inspired by earlier partial results [12-16], we performed a thorough analysis [11] of four-quark singularities in the Mandelstam variable $s$ due to the possible existence of tetraquark poles in Green functions. Its outcomes give reason to question the intrinsic consistency of investigating multiquark hadrons by means of traditional QCD sum rules [17,18]: the latter must be adapted to the challenge.

Insights [11] analogous to the above two-point case hold for, e.g., the three-point correlators of one tetraquark interpolating operator and two quark-bilinear currents, generating the amplitudes for transitions between a tetraquark and two ordinary mesons, if starting the derivation of the associated QCD sum rules from Feynman diagrams of the likewise tetraquark-phile type exemplified by Fig. 6.
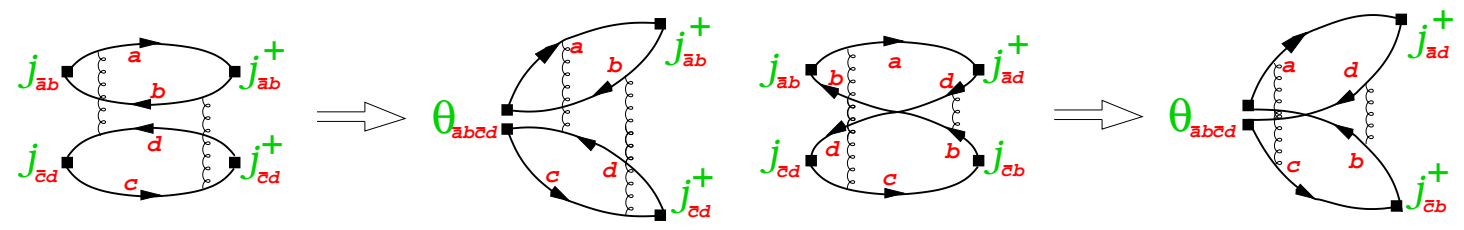

Figure 6: Flavour-preserving (left) and flavour-rearranging (right) Feynman diagrams contributing at lowest tetraquark-phile strong-coupling order $O\left(\alpha_{\mathrm{s}}^{2}\right)$ to correlators of four quark-bilinear currents $j$ and, by merging of just a single pair of currents $j$, to correlators of one tetraquark operator $\theta$ and two quark-bilinear currents $j$. 
Acknowledgements. D. M. and H. S. express gratitude for support under joint CNRS/RFBR Grant No. PRC Russia/19-52-15022. D. M. is grateful for support by the Austrian Science Fund (FWF), Project No. P29028.

\section{References}

[1] M. A. Shifman, A. I. Vainshtein, and V. I. Zakharov, Nucl. Phys. B 147 (1979) 385.

[2] K. G. Wilson, Phys. Rev. 179 (1969) 1499.

[3] W. Lucha, D. Melikhov, and S. Simula, Phys. Rev. D 79 (2009) 096011, arXiv:0902.4202 [hep-ph].

[4] W. Lucha, D. Melikhov, and S. Simula, J. Phys. G 37 (2010) 035003, arXiv:0905.0963 [hep-ph].

[5] W. Lucha, D. Melikhov, and S. Simula, Phys. Lett. B 687 (2010) 48, arXiv:0912.5017 [hep-ph].

[6] W. Lucha, D. Melikhov, and H. Sazdjian, Phys. Rev. D 96 (2017) 014022, arXiv:1706.06003 [hep-ph].

[7] W. Lucha, D. Melikhov, and H. Sazdjian, Eur. Phys. J. C 77 (2017) 866, arXiv:1710.08316 [hep-ph].

[8] W. Lucha, D. Melikhov, and H. Sazdjian, PoS (EPS-HEP 2017) 390, arXiv:1709.02132 [hep-ph].

[9] W. Lucha, D. Melikhov, and H. Sazdjian, Phys. Rev. D 98 (2018) 094011, arXiv:1810.09986 [hep-ph].

[10] L. D. Landau, Nucl. Phys. 13 (1959) 181.

[11] W. Lucha, D. Melikhov, and H. Sazdjian, Phys. Rev. D 100 (2019) 014010, arXiv:1901.03881 [hep-ph].

[12] S. Weinberg, Phys. Rev. Lett. 110 (2013) 261601, arXiv:1303.0342 [hep-ph].

[13] M. Knecht and S. Peris, Phys. Rev. D 88 (2013) 036016, arXiv:1307.1273 [hep-ph].

[14] T. D. Cohen and R. F. Lebed, Phys. Rev. D 90 (2014) 016001, arXiv:1403.8090 [hep-ph].

[15] L. Maiani, A. D. Polosa, and V. Riquer, J. High Energy Phys. 06 (2016) 160, arXiv:1605.04839 [hep-ph].

[16] L. Maiani, A. D. Polosa, and V. Riquer, Phys. Rev. D 98 (2018) 054023, arXiv:1803.06883 [hep-ph].

[17] M. Nielsen, F. S. Navarra, and S. H. Lee, Phys. Rep. 497 (2010) 41, arXiv:0911.1958 [hep-ph].

[18] R. M. Albuquerque et al., J. Phys. G 46 (2019) 093002, arXiv:1812.08207 [hep-ph]. 